
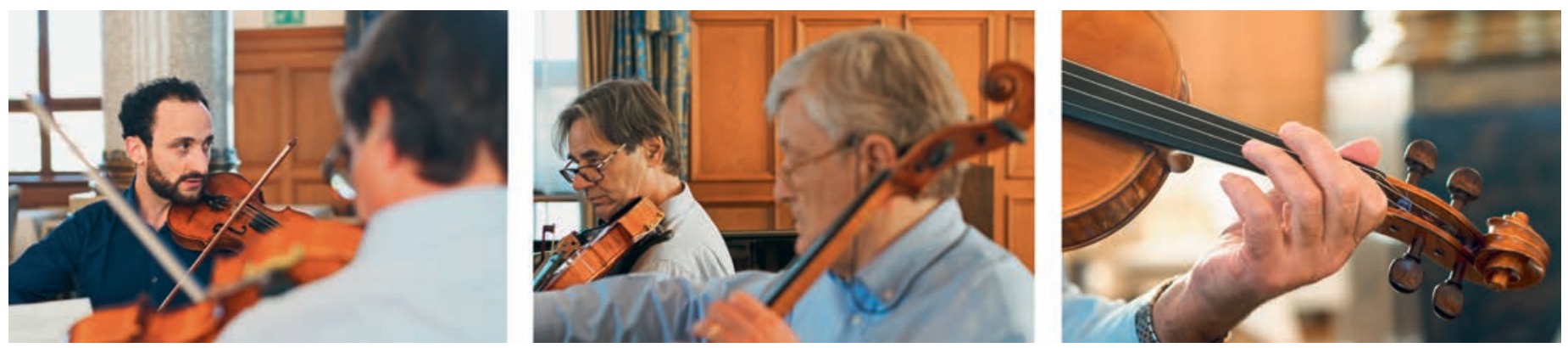

\title{
«Wir wollen Perlen ans Licht holen»
}

\section{Daniel Lüthi}

Freier Journalist und Fotograf, Medientrainer, Bern

Der Rahmen ist würdig: Die vier Herren üben im Belle-Epoque-Saal des Nobelhotels «Les Trois Rois» in Basel. Schöne Säulen, schönes Holz, schöne Leuchter - schönes Ambiente. Welch ein Kontrast zu einem sterilen Operationssaal, in dem sich zumindest drei der vier vor geraumer Zeit auch hätten treffen können. Denn der Pianist, der Cellist und der Bratschist sind vor allem Orthopäden, und erst in zweiter Linie Musiker. Der musikalische Leiter des illustren Quartetts ist der einzige Profimusiker - und gleichzeitig der Jüngste im Bunde.

1 https://www.youtube. com/ watch?v=KroL3KAoog8 (Suche unter Swiss Orthopaedic Quartet).
Im Jugendstilsaal sind grosse Konzentration und bisweilen auch etwas Anspannung auszumachen. Draussen fliesst ruhig der Rhein, wie immer. Das moderne Hochhaus der Pharmaindustrie am anderen Ufer ist gut sichtbar, gefühlsmässig aber weit weg. Gesprochen wird wenig, wie damals im Operationssaal auch. Heute geht es um Synkopen und Ritardandi, um Tempi-Wechsel und Harmonien, und es geht auch um den richtigen Einsatz. "Dieser A-Dur-Akkord ist ja kein Problem", sagt der musikalische Leiter. "Das Problem ist in meinem Kopf», sagt der Pianist, der sich vor seiner Pensionierung unter anderem mit einem Standardwerk in der Kinderorthopädie einen Namen gemacht hat.

Später, im Gespräch am grossen runden Tisch, äussert er sich zu dem, was seine beiden Leidenschaften, die Medizin und die Musik, verbindet. "Als Chirurg habe ich von der manuellen Geschicklichkeit profitiert, die ich mit meinem Klavierspiel immer wieder trainiert habe», erklärt er. «Es geht um die Sensorik in den Fingern und damit unter anderem um das Gefühl für das Gewebe.» Um die Frage zum Beispiel also, wie viel Druck jetzt gerade angesagt ist. «Könntest du an dieser Stelle bitte etwas leiser sein?», fragt der musikalische Leiter etwas später.
Vor kurzem hat das Orthopäden-Quartett ein Werk von Vinzenz Lachner geprobt und erstmals auf einer CD verewigt. ${ }^{1}$ Heute steht das Klavierquartett in a-Moll Opus 1 von Josef Suk auf dem Programm. Der tschechische Komponist lebte von 1874 bis 1935 und war der Schwiegersohn von Antonín Dvořák. Suks Musik, die jetzt den stilvollen Saal erfüllt, verbindet romantische Schwere mit temporeicher Leichtigkeit. Sie ist effektvoll und lebendig. Und wirkt - zum Teil auch technisch - recht anspruchsvoll.

So etwas kriegt einer nicht mit gelegentlichem Musizieren nach der Pensionierung hin. Er sei zum Glück in einer musikalischen Familie aufgewachsen und seinem Vater "unendlich dankbar», dass er ihn auch in diesem Bereich gefördert habe, erzählt Luzi Dubs, der nicht nur Orthopäde und Musiker, sondern auch Handballer ist. «Zeit», sagt er, «ist eine Frage des Einteilens, und des Lebensentwurfes. Wenn ich nicht TV schauen muss, kann ich eben beispielsweise ernsthaft ein Instrument spielen. Mein Cello hätte ich nie und nimmer vernachlässigen wollen.» Deshalb habe er auch nicht Unfallchirurg mit vielen Pikettdiensten sein wollen. Auch ihm sei die Musik praktisch in die Wiege gelegt worden, ergänzt Peppo Brandenberg, und 


\section{Das Swiss Orthopaedic Quartet SOQ}

Das SOQ wurde 2014 gegründet und besteht aus drei Orthopäden und einem Profigeiger. Dies sind die einzelnen Mitglieder:

\section{Ronny Spiegel, Violine}

Aufgewachsen in Winterthur. Profigeiger in Zürich, Mitglied diverser Ensembles, z.B. als Primgeiger des S-Ensembles in Zürich.

Prof. Dr. med. Fritz Hefti, Klavier

War bis vor sieben Jahren Ordinarius und Chefarzt für Kinderorthopädie an der Universitätskinderklinik beider Basel. Er ist Ehrenmitglied von Swiss Orthopaedics und war 1987 ASGFellow*

Dr. med. Luzi Dubs, Violoncello

War viele Jahre lang Orthopäde in Winterthur und von 1998 bis 2000 Präsident der SGOT (heute "Swiss Orthopaedics»). Er ist Ehrenmitglied von Swiss Orthopaedics und war 1985 ASG-Fellow*.

Dr. med. Josef E. Brandenberg, Viola

War bis vor vier Jahren Orthopäde in Luzern und von 2006 bis 2008 Präsident der SGOT (heute "Swiss Orthopaedics»). Er ist Ehrenmitglied von Swiss Orthopaedics und war 1986 ASG-Fellow*. Zurzeit ist er Präsident der FMCH.

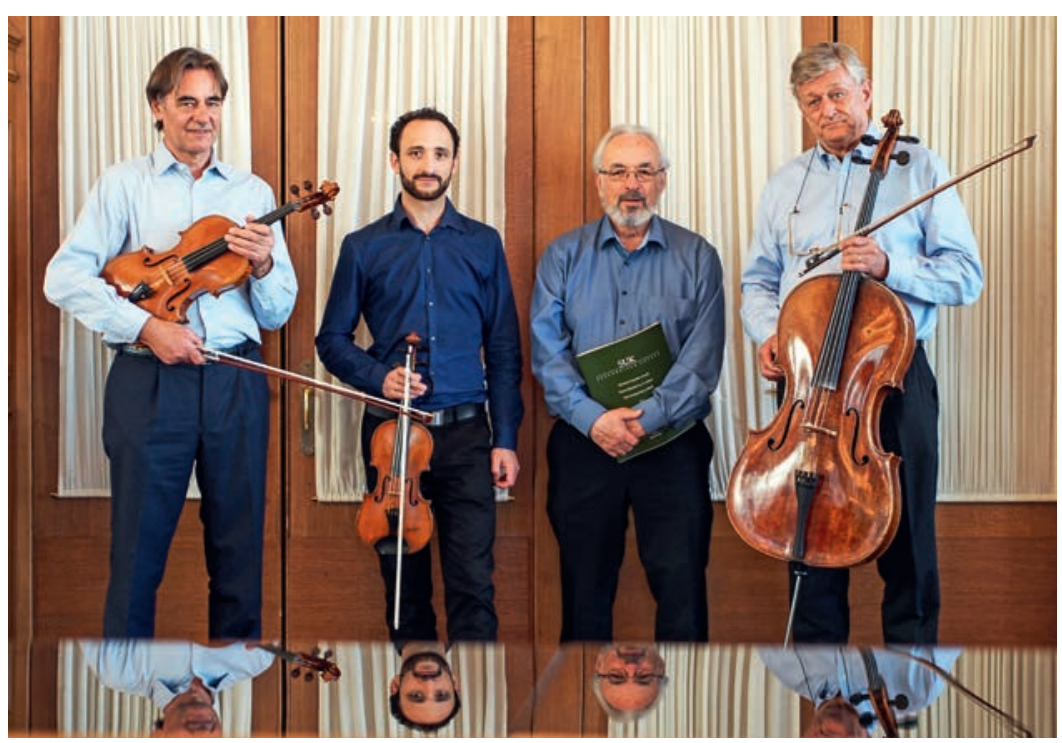

Beim ASG-Fellowship (Austrian-Swiss-German-Fellowship) handelt es sich um ein Reisestipendium der drei Orthopäden-Gesellschaften in Deutschland, Österreich und der Schweiz. Jährlich können zwei Deutsche, ein Österreicher und ein Schweizer gemeinsam nach England, Kanada und in die USA reisen und während rund zweier Monate bekannte Orthopäden-Zentren besuchen.. auch er habe immer versucht, dranzubleiben. «Wenn ich mich auf die Musik konzentriere, spüre ich meinen Puls», schwärmt er. «Da kann ich nicht mehr darüber nachdenken, was bei den TARMED-Tarifen schiefläuft.» Was hier geprobt wird, soll am 40-Jahr-Jubiläum der "ASG-Fellows»* nach dem traditionellen Spargelessen dargeboten werden. Einspielungen gibt es von diesem Werk schon, «deshalb werden wir es nur live spielen», erklärt Luzi Dubs, der Spiritus Rector des Quartetts. «Wir wollen uns ja nicht in Konkurrenz mit Profimusikern begeben. Wir wollen uns nicht profilieren. Es geht uns vor allem um die Förderung eines unbekannten musikalischen Repertoires. Wir wollen Perlen ans Licht holen.» Die Stimmung im Ensemble ist gut, allen ist der Teamgeist wichtig. Während des Spielens gehen kurze Blicke hin und her, die nonverbale Kommunikation ist wichtig, wie im Operationssaal bisweilen auch. "In der Medizin gibt es zu viele Einzelkämpfer», sagt einer der Mediziner. «Das ist in der Musik auch so», sagt der Profimusiker. Wie ist es für ihn, mit Nicht-Berufsmusikern zu üben und aufzutreten? Könnte das allenfalls seinem Ruf, seiner eigenen Karriere schaden? «Ich stehe voll hinter diesem Projekt», sagt Ronny Spiegel. «Ich schätze das Herzblut meiner Kollegen, und ich schätze es auch, meine Arbeit auf diese Weise zu reflektieren. Ich bewege mich gerne in verschiedenen Feldern.» Es ist ein eindrückliches Erlebnis, diese Mediziner in einem so anderen Feld zu beobachten. Das Temperament des Einzelnen wird spürbar, wenn er etwas besonders gut macht oder auch mal etwas verpatzt. "Die Bogenspannung ist wie eine Seelenspannung», sagt einer, "man gibt etwas von seinem Charakter preis.» Ein anderer ergänzt: «Technische Perfektion ist das eine. Das andere sind Schwingungen, Energien.» Und plötzlich wird unwichtig, wer was gesagt hat. Das Quartett ist nicht mehr ein Konglomerat von vier Einzelpersonen, sondern ein Ensemble. Und im Zentrum steht die Musik.

\section{Bildnachweis}

Fotos Daniel Lüthi
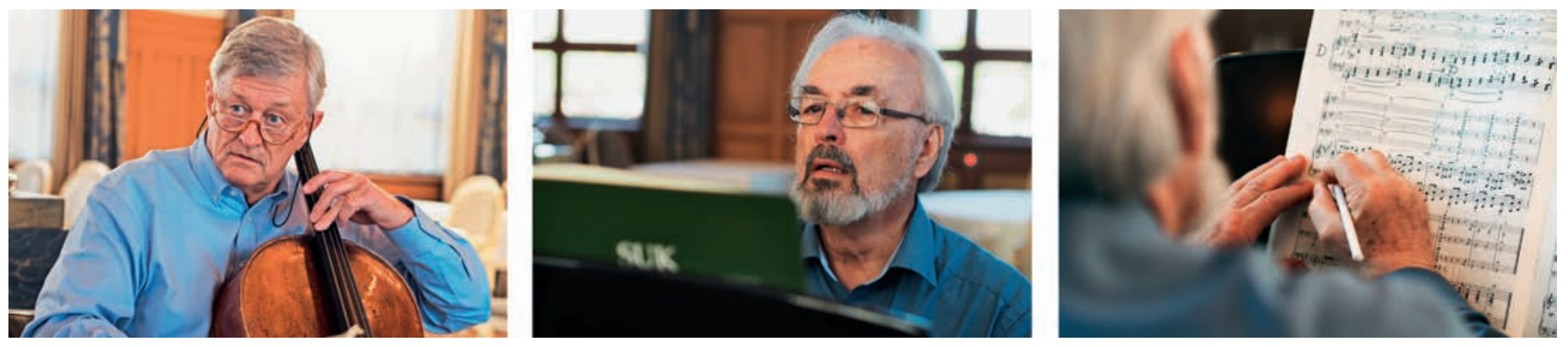